\title{
Out-of-Coverage Multi-Hop Road Safety Message Distribution via LTE-A Cellular V2V (C-V2V)
}

\author{
Lorenzo Gibellini and Maria Luisa Merani \\ Dipartimento di Ingegneria "Enzo Ferrari” \\ Università degli Studi di Modena e Reggio Emilia, Italy \\ Email: lorenzo.gibellini@gmail.com, marialuisa.merani@unimore.it
}

\begin{abstract}
This work investigates the performance of a multihop scheme for the dissemination of road safety messages on highway segments, employing the recently standardized LTE-A Cellular Vehicle-to-Everything (C-V2X) technology. In order to guarantee a seamless service in areas where cellular coverage is unavailable, vehicles directly communicate over the unlicensed ITS $5.9 \mathbf{~ G H z}$ frequency band, operating in accordance to Mode 4 of the C-V2X standard. The behavior of the proposed scheme reveals that the delivery of safety messages can successfully take place on a dedicated radio channel, as well as on a shared channel where periodic messages are broadcast at the maximum frequency foreseen by ETSI.
\end{abstract}

\section{INTRODUCTION}

Over the last few years, the evolution of Advanced Driver Assistance Systems (ADAS) is progressively shifting towards connected and automated driving. With the promise of improving road safety and traffic efficiency in future Intelligent Transportation Systems (ITS), evolved driving features like Cooperative Adaptive Cruise Control (CACC) and automated platooning will require real-time vehicle information exchange with the surrounding environment, encouraging the development of highly reliable and low-latency vehicular communication systems.

Consistently, research on Vehicle-to-Vehicle (V2V), Vehicleto-Infrastructure (V2I) and Vehicle-to-Pedestrian (V2P), collectively referred to as Vehicle-to-Everything (V2X), is stirring a great deal of interest from both telecommunications and automotive business players. For this reason, the European Telecommunications Standards Institute (ETSI) has defined a standard architecture for ITS [1], including the protocol specifications for two kinds of application-layer packets, namely, periodic Cooperative Awareness Messages (CAMs) [2], conveying vehicle status information (e.g., speed, position, heading), and event-triggered Decentralized Environmental Notification Messages (DENMs) [3], warning about potentially dangerous situations that might show up on the road.

The earliest studies on V2X for road safety have led to the release of the Dedicated Short Range Communications (DSRC) standard, that encompasses 802.11p [4] as its accesslayer solution. As an alternative, the Third Generation Partnership Project (3GPP) has recently introduced support to Cellular-based V2X (C-V2X) communications in Release 14 of the Long Term Evolution-Advanced (LTE-A) standards, completed in June 2017. Unlike DSRC, C-V2X promises a clear evolutionary path towards future standard releases within the $5 \mathrm{G}$ framework and relies on the existing cellular network infrastructure, thus removing the need to install RoadSide Units (RSUs) exclusively dedicated to V2I services. In addition to this, Mode 4 in Release 14 enables direct exchange of data among Vehicular User Equipments (V-UEs) that are in radio visibility, on the unlicenced $5.9 \mathrm{GHz}$ ITS frequency band.

At the time of this writing, $\mathrm{C}-\mathrm{V} 2 \mathrm{X}$ research outcomes are mostly confined to simulation studies, owing to: (i) the limited availability of commercial hardware platforms; (ii) the significant costs entailed by large-scale measurement campaigns in real traffic environments. The first simulative investigations appeared in literature very recently too: in [5], Masegosa et al. evaluated the reliability performance, measured in terms of Packet Reception Ratio (PRR), when broadcasting periodic CAMs in an urban traffic scenario through Mode 4 singlehop transmissions. In [6], the same authors investigated the reliability of single-hop CAM message delivery in a highway environment under different operating conditions (e.g., vehicle density, Modulation and Coding Scheme, CAM repetition rate). Unlike these previous studies, the current work proposes a distribution scheme for event-triggered DENM safety messages on roadways, that leverages on multi-hop communications and relies on the $\mathrm{C}-\mathrm{V} 2 \mathrm{~V}$ standard to address the out-of-coverage case. Moreover, it examines two alternative transmission schemes, that is, the adoption of a dedicated frequency channel for DENM messages, as well as the case where DENM packets compete with periodic CAM messages to gain access to radio resources along the multi-hop trajectory. The main contributions that this work puts forth can be summarized as follows:

- it substantiates the feasibility of DENM message delivery via multi-hop C-V2V on highways;

- it demonstrates that the end-to-end latency incurred by such packets is lower than $300 \mathrm{~ms}$ with probability 0.998 at a distance of $3500 \mathrm{~m}$ from the vehicle issuing the safety messages;

- it proves that a modest length repetition pattern of the DENM message on the first hop is sufficient to guarantee high reliability to the multi-hop delivery process, even in the presence of competing CAM traffic;

- it recognizes that for the proposed scheme, such reliability mainly depends of the effectiveness of the delivery on 
the very first hop.

Even though multi-hop packet dissemination in vehicular networks has been deeply researched in the past through analytical models [7] and numerical simulations [8][9], previous studies considered $802.11 \mathrm{p}$ as the primary V2V communication technology, whereas, to the best of our knowledge, this is the first work focusing on multi-hop DENM dissemination via $\mathrm{C}-\mathrm{V} 2 \mathrm{~V}$

The remainder of the current paper is organized in five Sections, where Section II briefly reviews PHY and MAC layer procedures for direct $\mathrm{V} 2 \mathrm{~V}$ communications as specified by 3GPP Release 14, placing the main emphasis on the distributed resource selection algorithm for out-of-coverage operation. Next, Section III illustrates the multi-hop safety message dissemination scheme for the relevant use case of highway deployment. Lastly, Section IV discusses the numerical results and Section V draws the conclusions.

\section{3GPP SIDELINK V2V}

As anticipated in the Introduction, Release $14 \mathrm{C}-\mathrm{V} 2 \mathrm{X}$ enables V-UEs to directly exchange data over the so-called sidelink radio interface, also termed the PC5 interface in LTEA jargon. More specifically, two new V2X-tailored sidelink operating modes have been added in Release 14, Mode 3 and Mode 4. At PHY layer, they both foresee the transmission on the unlicensed $5.9 \mathrm{GHz}$ ITS band, employing either $10 \mathrm{MHz}$ or $20 \mathrm{MHz}$ wide channels. Moreover, they adopt the SingleCarrier Frequency Division Multiple Access (SC-FDMA) transmission scheme, with two extra Demodulation Reference Signals being inserted within the frame pattern; this warrants an improved channel estimation capability at the receiver, even under significant Doppler shifts. Interestingly, whereas Mode 3 still relies on the cellular network to perform radio resource assignment, Mode 4 does not, therefore appearing as the ideal candidate for safety applications, required to flawlessly operate in out-of-coverage scenarios too.

In LTE-A Release 14, radio resources can be interpreted as organized along the grid that Fig.1 portrays, where $1 \mathrm{~ms}$ long subframes repeat along the horizontal time axis, whereas frequency resource blocks (RBs), $180 \mathrm{KHz}$ wide, appear on the vertical axis and represent the smallest resource unit that a vehicle can utilize for transmission. To accommodate the transmission of an entire data packet in the same subframe, RBs can be grouped in subchannels. In Mode 3 and 4, every data message is paired with its Sidelink Control Information (SCI), transmitted within the same subframe and conveying crucial information for the successful decoding of the data message at the receiver side. Whereas the size of a subchannel is not standardized, the SCI always occupies two consecutive RBs.

One major concern in sidelink $\mathrm{V} 2 \mathrm{~V}$ communications is the non-zero probability of air frame collisions, that occur when more vehicles simultaneously employ the same radio resources. In order to reduce/confine collisions, appropriate scheduling mechanisms have been envisioned. Under cellular network coverage, Mode 3 operation can be adopted, whereby

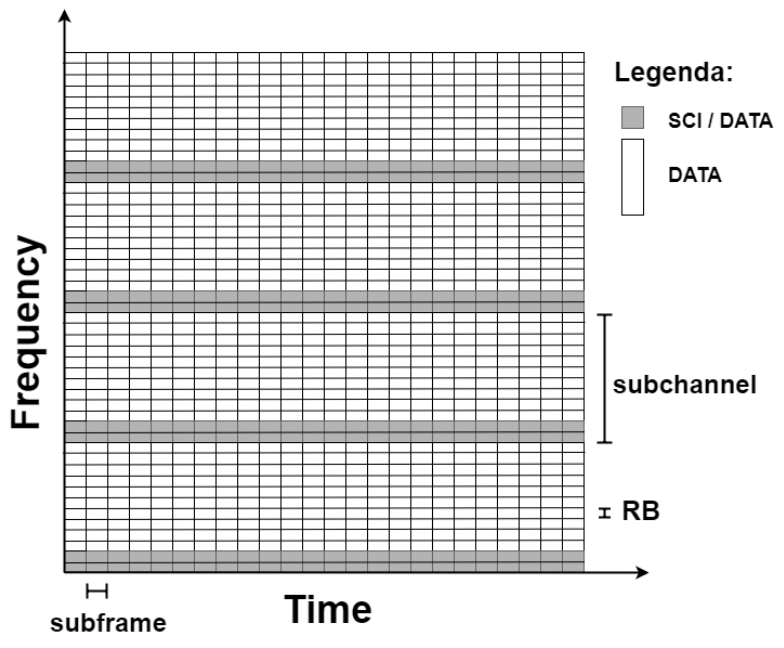

Fig. 1. Mode 3 and Mode 4 radio resource grid

radio resources are scheduled by the serving enodeB, that avoids resource assignment conflicts owing to a comprehensive view of all the attached V-UEs. Instead, out-of-coverage operation cannot rely on cellular network assistance. For this reason, Mode 4 adopts an autonomous resource selection mechanism that combines a channel sensing algorithm with a semi-persistent resource reservation procedure.

According to 3GPP TS 36.213 [10], the outcome of Mode 4 distributed scheduling is the selection of a single-subframe resource (SSR), that is defined as a set of adjacent resource blocks accommodating data and control transmissions during a subframe. Semi-persistent scheduling implies that, once selected, the same frequency resources will be periodically reused by the same V-UE for a number of $C_{\text {resel }}$ consecutive times, where $C_{\text {resel }}$ is the reselection counter, that is initially set to a random integer value. For periodic CAM beacons, the reservation interval $P_{\text {rsvp }}$ between two consecutive transmissions is set by higher layers to match the application-layer repetition rate (e.g., $100 \mathrm{~ms}$ ). When $P_{\text {rsvp }} \geq 100 \mathrm{~ms}$, the initial $C_{\text {resel }}$ is drawn from an integer uniform distribution in the $[5,15]$ interval. After each new transmission, $C_{\text {resel }}$ is decremented by one and, if it has not reached zero, the VUE stores a sidelink grant for next transmission. Therefore, when the V-UE MAC sublayer receives a request to transmit data, it first checks for the presence of a previously scheduled grant; if it does not find it (i.e., $C_{\text {resel }}$ has expired), it selects new resources with probability $1-P$, where $P \in[0,0.8]$ is configured by higher layers. Re-selection is also performed when the previously scheduled number of subchannels is not sufficient to serve the new data transmission.

As the goal of distributed scheduling is to have the V-UE choose an SSR so as to minimize the collision probability, the vehicle monitors the channel and stores its "history" during the last $1000 \mathrm{~ms}$, learning which radio resources are available from the reservation information conveyed by the received SCIs, as well as from power level measurements performed over the frequency subchannels. More specifically, 
the vehicle autonomously builds a set $S$ of Candidate Singlesubframe Resources (CSRs); at the very beginning, $S$ includes all the SSRs falling into the selection window, whose duration coincides with the maximum tolerable PHY-layer latency data messages can bear, also referred to as the Packet Delay Budget (PDB). After that, the V-UE identifies those resources that, based on the SCI previous receptions, have already been reserved by other vehicles. At this point, the V-UE examines the most recent measurements of the average Reference Signal Received Power (RSRP) observed over the frequency RBs constituting the already reserved CSRs and excludes from $S$ those CSRs that are reserved and display an average RSRP above a given threshold. Moreover, since 3GPP C$\mathrm{V} 2 \mathrm{~V}$ specifications assume half-duplex operation, the V-UE cannot simultaneously transmit and monitor the channel. So, if the V-UE was transmitting during subframes $t_{z}-j \cdot P_{\text {rsvp }}$, with $j=1,2, \ldots, 10$, all CSRs belonging to subframe $t_{z}$ are also excluded from $S$. The number of CSRs in $S$ has to amount to at least $20 \%$ of the resources within the selection window; otherwise, the procedure is repeated increasing the RSRP threshold by $3 \mathrm{~dB}$ at each iteration until the condition on the size of set $S$ is satisfied. Last step requires the CSRs in $S$ be sorted based on their average Sidelink Received Signal Strength Indication (S-RSSI), to exclude from $S$ the $80 \%$ of resources experiencing the highest average S-RSSI values. Finally, the actual transmission resource is randomly chosen from the residual CSR set.

\section{SAFety Message Delivery}

This Section illustrates the proposed multi-hop dissemination strategy that exploits Mode $4 \mathrm{C}-\mathrm{V} 2 \mathrm{~V}$ for safety message distribution in a highway scenario.

When traveling on the road, vehicles might encounter a series of potentially dangerous situations such as car accidents, unexpected fog banks, icy road segments. Exclusively relying on their on-board sensors and cameras, vehicles will become aware of the danger too late to proactively react to and avoid it. By contrast, our aim is to warn not only nearby, but also distant cars, allowing drivers to timely take corrective actions (slowing down, changing route). We concentrate on a highway scenario and given that highways also cross suburban and rural areas, where V-UEs are likely to experience intermittent cellular network connectivity, we assume the adoption of sidelink Mode 4. As highlighted in the previous Section, its distributed scheduling mechanism does not require the assistance of the operator network. We further make the hypothesis that one vehicle $\mathrm{V}-\mathrm{UE}_{S}$, the source, detects the occurrence of the dangerous event and correspondingly broadcasts the DENM safety message conveying the event type, its geographical location, the generation timestamp and the packet relevance area. Once generated, the message has to be quickly delivered to all vehicles that are approaching the event area, whereas vehicles that are moving away from it will not be interested in receiving the message; on a highway, this implies that the safety packet has to be disseminated downstream with respect to the direction of the traffic flow.
For both CAM and DENM messages, each applicationlayer packet is mapped into a single MAC-layer transport block (TB). As it has been envisioned in [11], DENMs are larger than CAMs; as a consequence, they do not fit the resources currently allocated to the source vehicle $\mathrm{V}-\mathrm{UE}_{S}$ for the transmission of its CAM messages, for the same

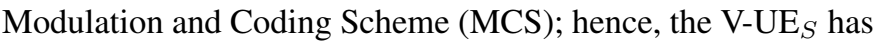
to break the former reservation and to select new resources. This will insignificantly affect the periodic CAM beaconing, as the latter will be interrupted for a very short time only.

In order to handle the transmission of the DENM packet, V$\mathrm{UE}_{S}$ next invokes the Mode 4 autonomous resource selection algorithm, assuming the duration of the selected window equal to the minimum PDB value indicated by 3GPP specifications for periodic beacons, namely, $P D B_{\min }=20 \mathrm{~ms}$ [12]. Unlike CAMs however, DENM generation is event-triggered, so reserving future resources is not only useless, but also harmful, as it deceives other vehicles; to avoid it, the source vehicle sets the reselection counter $C_{r e s e l}$ and the reservation period to zero.

With the purpose of improving the application-layer reliability (i.e., increasing as much as possible the Packet Reception Ratio, PRR, at different distances between the source and the destination vehicles), ETSI standards allow to repeat DENM transmissions for a preconfigured number of times [3]. In our scheme, DENM consecutive retransmissions can therefore take place; to avoid the broadcast storm problem however, only the source vehicle that originates the DENM message is permitted to repeat it, issuing $D E N M_{0}, D E N M_{1}, \ldots$, $D E N M_{K}$ packets and selecting new SSRs from scratch at every transmission. To this regard, we propose the application layer not to wait for the end of the current selection window before generating the next message copy; rather, a repetition is triggered $t_{r e p} \mathrm{~ms}$ after the previous, where $t_{r e p}<P D B$, with $t_{r e p}=\frac{P D B}{2}=10 \mathrm{~ms}$ in the current study. Owing to this choice, the selection windows for the transmission of two consecutive DENM copies partially overlap, thus reducing the inherent increase in latency that any repetition process introduces. Fig. 2 graphically exemplifies the choice we have performed.

More importantly, owing to the limited communication range of a single transmission, rebroadcasting by other vehicles is necessary to deliver the DENM over distances whose order of magnitude is a few kilometers.

The proposed multi-hop delivery process mandates that message re-broadcasting by a vehicle that receives the DENM will be performed, provided the two conditions reported below are met:

1) the relaying V-UE is within the DENM relevance area. For the highway scenario, this is the road segment preceding the event position;

2) the relaying V-UE has received the DENM packet for the first time.

From the implementation viewpoint, the two conditions can be readily checked. To verify the first, the event location and relevance area fields within the DENM packet have to be 


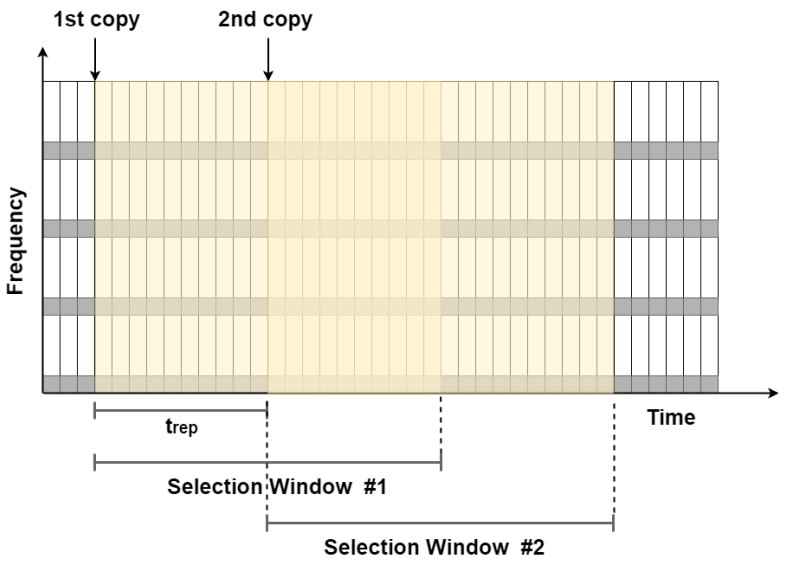

Fig. 2. Proposed Selection Window overlapping for two redundant DENM transmissions

compared against the current vehicle position. Checking the second requires the vehicle to keep track of the reception history for previous DENMs; this can be easily achieved storing the unique identifiers of the received packets. When the above conditions are both satisfied, they force the right direction on multi-hop message propagation and also significantly contribute to confine the broadcast storm problem. Indeed, multiple copies of the same DENM message might be received by the vehicle; if rebroadcasting were performed after each reception event, the number of generated packets would very quickly rise, inevitably leading to channel congestion.

\section{A. Key Performance Indicators}

When considering any multi-hop scheme for safety message dissemination, a relevant performance indicator is the delay that the message incurs traveling over a given distance. More accurately, we define such end-to-end latency, $\tau_{e 2 e}$, as the interval elapsing from the point in time when the safety message is generated by the application-layer module on the originating vehicle, up to the point in time when it is correctly delivered to the peer application-layer entity on the receiving vehicle. In the examined scenario, where more vehicles contribute to the dissemination of the safety message, multiple copies of it are likely to be received by the same vehicle; for $\tau_{e 2 e}$ estimation, only the first reception is considered, as it is the only relevant event in the end-to-end process of message delivery.

Given that:

- $k$ is the discrete random variable (RV) representing the index of the first DENM message being received at distance $d$;

- $t_{r e p}$ is the application-layer DENM repetition interval;

- $N(d)$ is the RV representing the number of hops between the originating and the receiving vehicle at distance $d$;

- $\tau_{i}$ is the rv representing the access latency on the $i$-th hop that the safety message incurs in, then

$\tau_{e 2 e}$ can be expressed as

$$
\tau_{e 2 e}(d)=k \cdot t_{r e p}+\sum_{i=1}^{N(d)}\left(\tau_{i}+\delta_{i}\right)
$$

where $\delta_{i}$ is the application-layer processing delay introduced before the rebroadcasting on the $i$-th hop takes place.

The Packet Reception Ratio (PRR) is the additional performance metric to be considered in order to assess transmission reliability. For a road scenario, PRR is defined as the fraction of vehicles that correctly receive the safety message within a given geographical area.

\section{NumericAl RESUlTS}

In this Section, we present the simulation setup and the results, in terms of $\tau_{e 2 e}$ and $P R R$, for the proposed safety message dissemination scheme when it is employed on a linear highway segment. To this aim, a custom C-V2X simulation tool has been developed within the ns-3 network simulator by significantly enhancing the D2D module provided in [13].

\section{A. Simulation Setup}

A regular vehicular network topology has been considered in all simulations; as in [6], communications are assumed to occur within a one-dimensional platoon, that in the current study is constituted by $M=200$ vehicles following the same regular motion pattern, that is, the same straight-line trajectory. Each vehicle is at a constant distance $D=20 \mathrm{~m}$ from the vehicle in front of it, as Fig.3 shows. Since the out-of-coverage case is addressed, vehicles communicate via sidelink Mode 4 with autonomous resource selection. Transmissions occur on a $10 \mathrm{MHz}$ channel at $5.9 \mathrm{GHz}$ with a transmission power of $23 \mathrm{dBm}$; the receiver sensitivity is set to $-90.4 \mathrm{dBm}$, that represents the maximum value assumed by 3GPP [14]. As suggested by TR 36.885 [15] for the freeway scenario, the propagation loss has been modeled by the Winner II + B1 Line-of-Sight (LOS) empirical model [16]. Moreover, the Nakagami distribution has been adopted for modeling fast fading, with the shape parameter $m$ set as in [17] for highway V2V environments. The received average Signal-toInterference-plus-Noise Ratio (SINR) for each received TB is computed on the basis of the received power, of the noise power spectral density (set equal to $-174 \mathrm{dBm} / \mathrm{Hz}$ ) and of the interference level. The SINR value is finally mapped into a PHY-layer packet error probability based on the BLock Error Rate (BLER) curves provided in [18].

As in [5], the available $10 \mathrm{MHz}$ bandwidth is partitioned into $N_{s u b C H}=4$ subchannels, each constituted by $n_{\text {subCH size }}=12$ Resource Blocks (RBs). TBs carrying CAMs and DENMs will be 190 Byte and 1000 Byte long, respectively. Therefore, assuming QPSK modulation with 0.7 Code Rate, CAMs will occupy one subchannel, whereas DENMs will require the entire channel bandwidth (i.e., all the four available subchannels). In our simulations, each vehicle begins to broadcast periodic CAMs at an instant randomly picked from a uniform distribution within the $[100,300] \mathrm{ms}$ interval after the simulation start: this avoids undesirable synchronization effects; to ensure that the Mode 4 selection algorithm operates in the "true regime" condition, the originating V-UE issues the safety message and its copies $2000 \mathrm{~ms}$ after the 


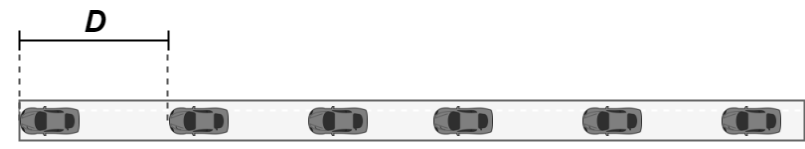

Fig. 3. Platoon network topology

simulation starts. Moreover, the application-layer processing delay $\delta_{i}$ is constant and equal to $3 \mathrm{~ms}$.

The performance of the multi-hop DENM dissemination scheme has been studied both in the absence and in the presence of concurrent CAM traffic. In the former case, DENM broadcast relies on a dedicated $10 \mathrm{MHz}$ channel, while in the latter, shared channel case, DENM messages share transmission resources with periodic CAM packets, whose transmission coverage is confined to one hop and whose repetition period is assumed to be $100 \mathrm{~ms}$, corresponding to the maximum rate envisioned by ETSI [2]. Although this last approach is more efficient from a resource utilization point of view, it might entail a non-negligible collision probability, that causes the decrease of $P R R$. Our numerical study aims at understanding how relevant this effect is and if it prohibits or allows the coexistence of CAM and DENM traffic on the same frequency band.

\section{B. Simulation Outcomes}

Under the previous assumptions, the newly developed ns$3 \mathrm{C}-\mathrm{V} 2 \mathrm{X}$ modules have been employed to estimate the endto-end latency $\tau_{e 2 e}$ and the packet reception ratio $P R R$ of the proposed multi-hop safety dissemination strategy. For the shared channel configuration, the choices of one single DENM transmission and one transmission corroborated by one or two repetitions have been examined.

As eq.(1) evidences, the end-to-end latency at a given distance $d$ between the originating and the receiving vehicle is tightly related to the number of transmission hops $N(d)$ traveled by the DENM safety message. Accordingly, Fig.4 reports the average value of $N(d), \overline{N(d)}$, as a function of $d$, as well as the corresponding t-Student $95 \%$ confidence intervals, obtained from 1000 uncorrelated simulation runs, so as to guarantee sound results. The width of the confidence intervals is fairly large and this is intrinsic to the multihop delivery process, as pointed out in [8]. Moreover, it can be observed that $\overline{N(d)}$ exhibits a linear dependency on $d$; also note that the slope is a little steeper for the shared channel configuration (solid line), an outcome explained by the higher collision probability the presence of CAM message transmissions induces.

Fig.5 shows a similar behavior for the average end-to-end latency $\overline{\tau_{e 2 e}}$ and reveals that for the shared channel case $\overline{\tau_{e 2 e}}$ increases with $d$ according to a larger slope too. Also note that the average end-to-end latency of the shared channel configuration case modestly increases when the DENM is repeated once (i.e., 2 consecutive transmissions) or twice (i.e., 3 transmissions), as it happened for the average number of hops. This is explained by the fact that some vehicles might need to wait for the second or third transmission before receiving the first packet copy. Such behavior is confirmed by the $\tau_{e 2 e}$ Cumulative Distribution Function (CDF) reported in Fig.6, where the performance of the dedicated and shared channel schemes are examined for three different values of the distance, $d=1500,2500$ and $3500 \mathrm{~m}$, and for either 0 , 1 or 2 repetitions of the safety message. Even though the dedicated channel configuration always provides the best latency performance, it is significant to outline that the shared channel solution with 2 repetitions guarantees $\tau_{e 2 e}$ values lower than $300 \mathrm{~ms}$ at distance $d=3500 \mathrm{~m}$ with probability 0.998. It can then be concluded that in the examined scenario, sufficiently low end-to-end latency values can be achieved even under the shared channel configuration, allowing drivers ample response times.

Next, Fig.7 shows the average DENM Packet Reception Ratio $\overline{P R R}$ as a function of $d$. Interestingly, DENM reliability does not depend on $d$. This behavior is explained observing that in the proposed dissemination strategy, the first hop plays a crucial role and dictates the overall performance. Indeed, unsuccessful packet delivery is more likely to occur within this first hop than in the next ones, where more vehicles receive the message and relay it, guaranteeing spatial diversity to the dissemination process. Therefore, DENM repetitions by the source vehicle serve the purpose of improving the robustness of first-hop transmission, increasing the $\overline{P R R}$ value and decreasing its confidence intervals. Numerically, when a single DENM transmission is performed in the shared channel case, $\overline{P R R}$ does not exceed 0.96 ; in the presence of redundant transmissions, reliability improves and the $\overline{P R R}$ achieves values very close to 1 with only two retransmissions. The width of the $95 \%$ confidence intervals is also significantly reduced.

Overall, this behavior suggests that it is not mandatory to reserve a $10 \mathrm{MHz}$ channel exclusively for DENM transmissions. Rather, a modest number of application-layer message repetitions by the originating vehicle are sufficient to provide a satisfactory PRR performance without significantly impairing the end-to-end latency or penalizing CAM periodic transmissions. Regarding this last aspect, we stress that the sporadic DENM transmissions that we have inserted within the overall traffic flow among V-UEs have nearly no effect on the performance experienced by CAMs, making of no relevance to investigate their figures of merit.

\section{CONCLUSIONS}

This work has investigated the feasibility of a multi-hop scheme for delivering safety messages built on top of $\mathrm{C}-\mathrm{V} 2 \mathrm{~V}$ Mode 4 standard, so as to guarantee its operation in out-ofcoverage areas. It has demonstrated the effectiveness of the proposed solution, and has revealed that reserving a $10 \mathrm{MHz}$ channel exclusively for DENM transmissions is not mandatory. Rather, a low number of DENM message repetitions confined within the first hop guarantees a high PRR to DENM packets in the configuration where DENM and CAM messages share 


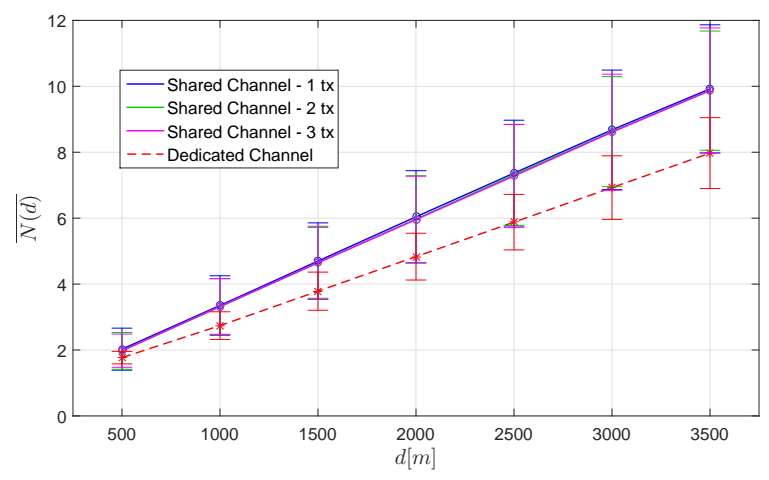

Fig. 4. Average number of hops to first DENM reception

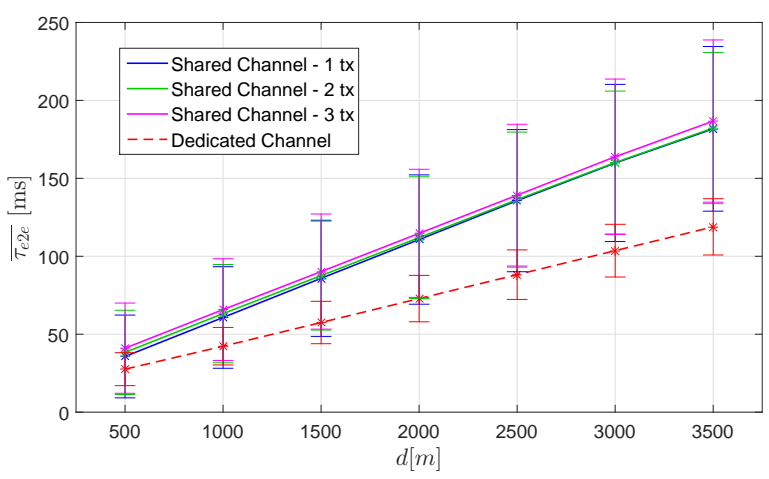

Fig. 5. Average DENM end-to-end latency

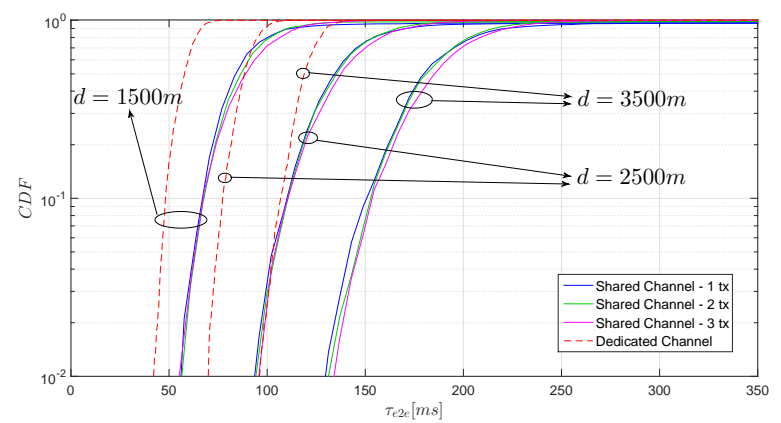

Fig. 6. CDF of DENM end-to-end latency

the same frequency band, at the cost of an irrelevant increase in the end-to-end delay they undergo.

\section{REFERENCES}

[1] ETSI EN 302665 V1.1.1, "Intelligent Transport Systems (ITS); Communications Architecture," Sept. 2010.

[2] ETSI EN 302 637-2 V1.3.2, "Intelligent Transport Systems (ITS); Vehicular Communications; Basic Set of Applications; Part 2: Specification of Cooperative Awareness Basic Service,” Nov. 2011.

[3] ETSI EN 302 637-3, v.1.2.2, "Intelligent Transport Systems; Vehicular Communications; Basic Set of Applications; Part 3: Specification of Decentralized Environmental Notification Basic Service," Nov. 2014.

[4] IEEE Std 802.11p-2010, "IEEE Standard for Information technologyLocal and metropolitan area networks- Specific requirements- Part 11: Wireless LAN Medium Access Control (MAC) and Physical Layer (PHY)

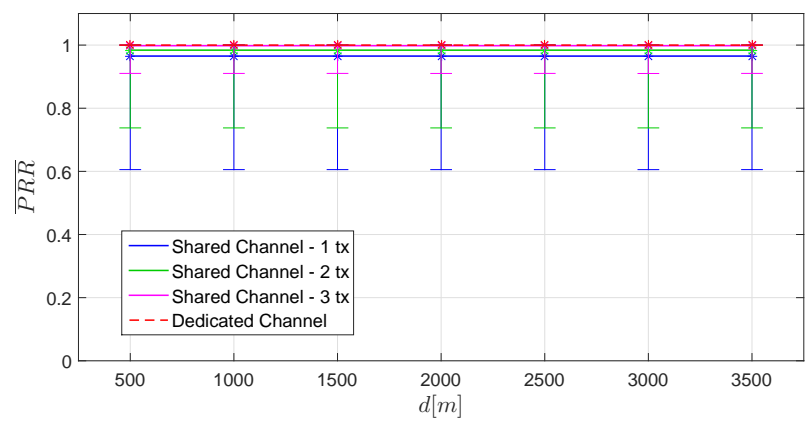

Fig. 7. Average DENM Packet Reception Ratio (PRR)

Specifications Amendment 6: Wireless Access in Vehicular Environments".

[5] R. M. Masegosa and J. Gozalvez, "System Level Evaluation of LTE-V2V Mode 4 Communications and Its Distributed Scheduling," 2017 IEEE 85th Vehicular Technology Conference (VTC Spring), 4-7 June 2017, Sydney, NSW, Australia.

[6] R. M. Masegosa and J. Gozalvez, "LTE-V for Sidelink 5G V2X Vehicular Communication," IEEE Vehicular Technology Magazine, December 2017.

[7] Y. Hu, H. Li, Z. Chang and Z. Han, "End-to-End Backlog and Delay Bound Analysis for Multi-Hop Vehicular Ad Hoc Networks," IEEE Transactions on Wireless Communications, vol. 16, no. 10, Oct. 2017.

[8] A. Mouradian and V. V'eque, "Dissemination Strategies in Realistic V2V Highway Networks: the Madrid Trace Case," 2016 Wireless Days (WD).

[9] S. Ucar, S. C. Ergen and O. Ozkasap, "Multihop-Cluster-Based IEEE 802.11p and LTE Hybrid Architecture for VANET Safety Message Dissemination," IEEE Transactions on Vehicular Technology, vol.65, no.4 Apr. 2016.

[10] 3GPP TS 36.213 V14.4.0, "Physical Layer Procedures (Release 14)", Sept. 2017.

[11] 3GPP TR 22.885 V14.0.0, "Study on LTE support for Vehicle to Everything (V2X) services (Release 14)", Dec. 2015

[12] 3GPP TS 36.321 V14.4.0, "Evolved Universal Terrestrial Radio Access (E-UTRA); Medium Access Control (MAC) protocol specification ( $R e-$ lease 14),' Sept. 2017.

[13] R. Rouil, F. J. Cintron, A. B. Mosbah, and S. Gamboa, "Implementation and Validation of an LTE D2D Model for ns-3," in Proceedings of the 2017 Workshop on ns-3, Porto, Portugal, June 2017 (WNS3 2017), doi: http://dx.doi.org/10.1145/3067665.3067668

[14] 3GPP TR 36.785 V14.0.0, "Vehicle to Vehicle (V2V) services based on LTE sidelink; User Equipment (UE) radio transmission and reception (Release 14)", Oct. 2016.

[15] 3GPP TR 36.885 V14.0.0, "Technical Specification Group Radio Access Network; Study on LTE-based V2X Services; (Release 14)," Jun. 2016.

[16] IST - 4-027756 WINNER II D.1.1.2 V.1.2, "WINNER II Channel Models".

[17] H. Schumacher and H. Tchouankem, "Highway propagation modeling in VANETS and its impact on performance evaluation," 2013 10th Annual Conference on Wireless On-demand Network Systems and Services (WONS), Banff, AB, Canada, 2013, pp. 178-185.

[18] Huawei - HiSilicon, 3GPP R1-160284, "DMRS enhancement of V2V," 3GPP TSG RAN WG1 Meeting \#84, St. Julian's, Malta, 15th-19th February 2016 